\title{
Comparison of Dual Task and Task Oriented Training Programme on Gait in Chronic Stroke
}

\author{
Amandeep Singh ${ }^{1,2, ~ *, ~ D a v i n d e r ~ K a u r ~}{ }^{1,2}$, Rajneet Kaur Sahni ${ }^{1,2}$, Simran Grewal ${ }^{1,2}$ \\ ${ }^{1}$ Physiotherapy Department, All Saints Institute of Medical Science and Research, Ludhiana, India \\ ${ }^{2}$ Baba Farid University of Health Sciences, Faridkot, India
}

Email address:

adsingh88@gmail.com (A. Singh), davinderjandu90@gmail.com (D. Kaur), rajneetsahni86@gmail.com (R. K. Sahni), simrangrewall6only@gmail.com (S. Grewal)

${ }^{*}$ Corresponding author

\section{To cite this article:}

Amandeep Singh, Davinder Kaur, Rajneet Kaur Sahni, Simran Grewal. Comparison of Dual Task and Task Oriented Training Programme on Gait in Chronic Stroke. International Journal of Neurologic Physical Therapy. Vol. 5, No. 2, 2019, pp. 42-50.

doi: 10.11648/j.ijnpt.20190502.13

Received: August 30, 2019; Accepted: September 25, 2019; Published: October 20, 2019

\begin{abstract}
Stroke is the major cause of disability and handicap in adults and it usually results in some degree of muscle weakness. Daily living requires balance and walking ability while performing tasks. Several factors affect the functional independence in post stroke period in which gait plays a significant role. 30 subjects of age group 45-60 years were selected by purposive sampling and informed consent was taken. The subjects were divided into 2 groups A and B of 15 each. Both the groups received conventional physiotherapy as muscle strengthening of affected lower limb and balance training. Group A received dual task training and Group B received task oriented training for 8 weeks. Pre test and post test data for gait parameters (gait speed, cadence, step length, stride length) were obtained by using 10 meter walk test respectively. Unpaired' $t$ ' test was applied at the $\mathrm{p}<0.05$ for the comparison within and between the groups for the variable Gait Parameters(i.e gait speed, cadence, step length, stride length). The values of gait parameters between the groups were significant which showed that task oriented training is more effective than dual task training to improve gait in chronic stroke.
\end{abstract}

Keywords: Stroke, Dual Task Training, Task Oriented Training, Gait, 10-Meter Walk Test

\section{Introduction}

Stroke is an acute onset of neurological dysfunction due to an abnormality in cerebral circulation with resultant signs and symptoms that corresponds to involvement of focal areas of the brain. World Health Organization (WHO) classified stroke as "neurological deficit which must persist for at least 24 hour duration. [7] Stroke is the third most commonest cause of death in India according to the statistics. [1] Approximately 80 percent of strokes are due to ischemic cerebral infarction and 20 percent to brain hemorrhage. [2] An acute space occupying lesion which compresses, displaces and disrupts the surrounding tissue which causes an increase in intracranial pressure and may lead to herniation which ultimately resulting in edema formation, aggravation of the intracranial pressure rise, and risk for herniation. [3] In post stroke patients, the function of cerebral cortex becomes impaired, while that of the spinal cord is preserved. The complex interactions of the neuromusculoskeletal system should be considered when selecting and developing treatment methods that should act on the underlying pathomechanisms causing the disturbances. The spinal cord generates human walking, and the cerebral cortex makes a significant contribution in relation to voluntary changes of the gait pattern. [4] Movement involves not only motor skills, but is also reliant on sensory and cognitive systems. There is also evidence that some older people stop walking when they talk and that this phenomenon predicts susceptibility to falling. [5] A variety of physiotherapy interventions improve functional outcomes, even when applied late after stroke. These findings challenge the concept of a plateau in functional recovery of patients who had experienced stroke and should be valued in planning community rehabilitation services. [6] Trunk rehabilitation exercises, balance training, muscle strengthening is essential for post stroke patients for 
recovery of balance and improvement in gait and is an important component. [7] Many therapeutical approaches for rehabilitation of gait are useful after stroke. Out of which dual task (DT) training and task oriented training programme are being used. Cognitive-motor and motor dual tasks play important roles in daily life: walking while talking, using a mobile phone, carrying a bag or watching traffic. It has been suggested that dual task training might have greater efficacy for improving dual task performance compared to single task training. [8, 9] Gait related dual task deficits persist in community dwelling stroke survivors many months after discharge from rehabilitation. [10] Task oriented training involves a variety of practices to help patients derive optimal control strategies for solving motor problems. During task oriented training, many types of movement are practiced, to limit compensatory movements and increase adaptive movements. [11] It is a method which focuses on specific functional tasks associated with the musculoskeletal and neuromuscular systems and gait related tasks are practiced using a functional approach. [12] Task oriented training induce greater improvement in walking competency in people with stroke. [13]

Achievement of community ambulation after stroke is critical for active participation in everyday activities preventing social isolation and depression and enhancing quality of life. Despite initial improvements in motor function in individuals early post stroke, patients often demonstrate a "plateau," or deceleration of motor recovery in the chronic stages. Several previous studies have proven that dual task training and task oriented training improve the gait through improvement in gait speed, cadence, step length, stride length in stroke patients. But till date no evidence is available on the basis of peer review of articles which determine that out of dual task training and task oriented training which is more beneficial. The current study focus is to determine the most effective training out of above mentioned protocols for improving gait in chronic stroke.

\section{Main Body}

\subsection{Material and Methods}

Type of study is quasi experimental type of study. Samples were selected through Purposive Sampling. Subjects were taken from hospitals\& clinics in \& around Ludhiana. 30 subjects both males and females with age group between 45 60 years who were having stroke for at least 6 months and who were able to perform 10-Meter Walk Test. Mini -Mental State Examination (MMSE) minimum score 24 and Modified Ashworth Scale (MAS): Grade 1+, 2 were taken as samples. Subjects with Perceptual disorders, Recurrent stroke having past or present neurologic disorder other than stroke. Subjects with visual and auditory impairment or with the history of any orthopedic conditions interfering with gait. Subjects with Sensory impairments involving lower limb and Subjects withCardiovascular disorders and Respiratory disorders were excluded from the study.

\subsection{Procedure}

30 subjects with stroke were selected and divided into two groups. Group A and Group B of 15 subjects in each. Ethical and informed consents were obtained. The subjects were assessed and baseline measurement were taken using the ink foot-print record method before commencement of the therapy, by measuring the gait parameters i.e. step length, stride length, gait speed, cadence on a 10 meter walk way with a plain chart paper on its surface. Patients were instructed to step on an ink water and were asked to walk on chart sheet prior two steps were taken on ground. The footprints from the sole of the feet were produced on the paper as the patients walked from one end of the walkway to the other. The measurement of step length and stride length were taken. [14] The subjects were instructed to walk $14 \mathrm{~m}$ at a comfortable speed and were timed using a stop watch over middle $10 \mathrm{~m}$ to measure gait speed. [15] For the measurement of cadence, patients were instructed to walk for 1 minute and step were recorded.

Group A included 15 subjects who were given dual task training. In this conventional physiotherapy as muscle strengthening of affected lower limb and balance training were given to the patient prior to dual task training for 15 minutes.

Dual task training group were instructed to perform motor and cognitive dual task gait training (MCDGT) while walking on floor. In motor dual task gait training (MDGT) patients were instructed to perform 5 types of motor dual tasks. The tasks were 'tossing up and catching a ball', 'rehanging loops on different hooks', 'doing up buttons after unbuttoning', and 'holding a glass of water without spilling it', 'receiving and returning a glass of water'. [16] In cognitive dual task gait training (CDGT) patients were instructed to perform 5 types of cognitive dual tasks. The tasks were 'discerning colours', 'mathematical subtraction', 'verbal analogical reasoning', 'spelling words backward', 'counting backward". Duration of dual task training for both cognitive task and motor task was 30 minutes. Each task was practiced for 3 minutes a session, 3 sessions per week for 8 weeks.

Group B included 15 subjects who were given task oriented training. In this conventional physiotherapy as muscle strengthening of affected lower limb and balance training were given prior to task oriented training for 15 minutes.

Task oriented training group were instructed to perform10 tasks. These were (1) step ups (stool of constant step height of $15 \mathrm{~cm}$ i.e 6" (length18" and breadth 13"), (2) balance beam, (3) kicking a ball, (4) stand up and walk, (5) obstacle walking, (6) tandem walk, (7) walk and carry (any object), (8) speed walk, (9) walk backwards, and (10) stairs. Duration of task oriented training is 30 minutes. Each task was practiced for 3 minutes a session, 3 sessions per week for 8 weeks.

Post test data measurement were taken using the ink footprint record method after commencement of the therapy, by 
measuring the gait parameters. The data was collected, compiled and analyzed.

\section{Results}

Data was meaningfully assorted through calculation of Mean and Standard Deviation. Later on Paired't' test was applied for comparison within Group A and Group B for Gait Parameters i.e gait speed, cadence, step length, stride length. Thereafter unpaired 't'test was applied for comparison between Group A and Group B for gait parameters. The level for Significance was fixed at $\mathrm{p}<0.05$.

Table 1 and Figure 1 shows UnPaired ' $t$ ' test result of Gait Speed between Group A and B.. The Mean \pm SD value for Pre test of Gait speed of Group A was $0.37 \pm 0.104$ and pre test of Group B was $0.37 \pm 0.119$. The Mean and SD value for Post test of Gait Speed of Group A was $0.52 \pm 0.140$ and Post test of Group B was $0.63 \pm 0.126$. The ' $t$ ' value for Pre test comparison between Group A and Group B was 0.114, which was statistically non significant, at $p>0.05$ and Post test comparison between Group A \& Group B was 2.276, which was Statistically Significant, at $\mathrm{p}<0.05$.

Table 2 and Figure 2 shows UnPaired ' $t$ ' test result of Cadence between Group A and B.. The Mean \pm SD value for Pre test of Cadence of Group A was 57.07 \pm 21.674 and pre test of Group B was $61.33 \pm 23.454$. The Mean and SD value for Post test of Cadence of Group A was $80.93 \pm 14.916$ and Post test of Group B was $92.40 \pm 10.418$. The ' $t$ ' value for Pre test comparison between Group A and Group B was 0.517, which was statistically non significant, at $\mathrm{p}>0.05$ and Post test comparison between Group A \& Group B was 2.441, which was Statistically Significant, at $p<0.05$.

Table 3 and Figure 3 shows UnPaired ' $t$ ' test result of Step Length between Group A and B.. The Mean \pm SD value for Pre test of Step Length of Group A was 7.64 \pm 2.180 and pre test of Group B was $8.08 \pm 2.562$. The Mean and SD value for Post test of Step Length of Group A was 12.14 \pm 1.732 and Post test of Group B was 13.69 \pm 1.880 . The ' $t$ ' value for Pre test comparison between Group A and Group B was 0.507, which was statistically non significant, at $\mathrm{p}>0.05$ and Post test comparison between Group A \& Group B was 2.353, which was Statistically Significant, at $\mathrm{p}<0.05$.

Table 4 and Figure 4 shows UnPaired ' $t$ ' test result of Stride Length between Group A and B. The Mean \pm SD value for Pre test of Stride Length of Group A was 18.62 \pm 4.008 and pre test of Group B was 18.76 \pm 3.686 . The Mean and SD value for Post test of Stride Length of Group A was $24.51 \pm 4.137$ and Post test of Group B was 28.78 \pm 2.342 The ' $\mathrm{t}$ ' value for Pre test comparison between Group A and Group B was 0.100 , which was statistically non-significant, at p $>0.05$ and Post test comparison between Group A \& Group B was 3.476, which was Statistically Significant, at $\mathrm{p}<0.05$.

Table 5 and Figure 5 shows UnPaired ' $t$ ' test result of Age between the Group A and Group B. The mean \pm SD value for Group A was 53.53 \pm 3.681 and for Group B was 53.13 \pm 4.103 . The ' $t$ ' value for comparison of Age between Group A and Group Bwas 0.281, which was Statistically non Significant, at $\mathrm{p}>0.05$.

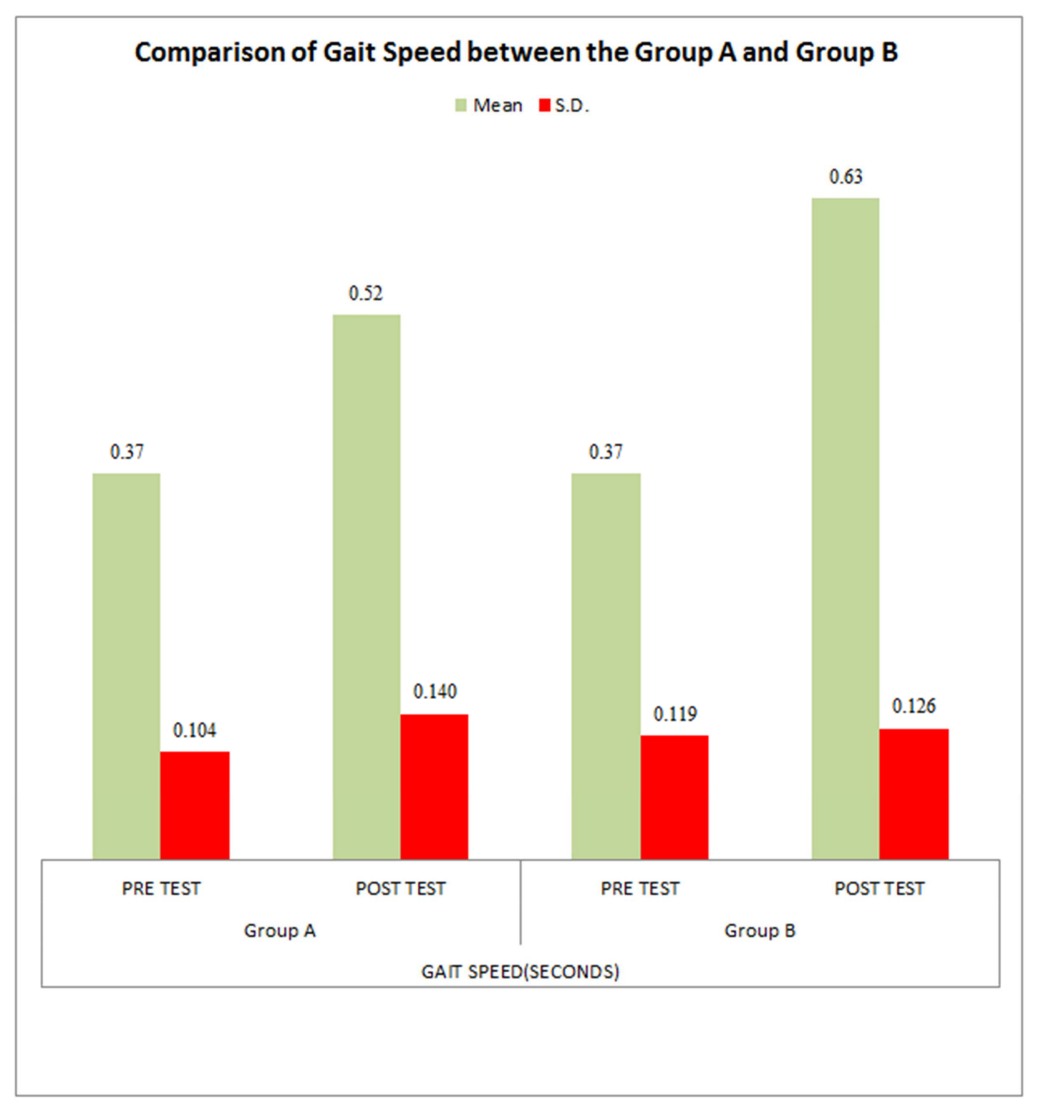

Figure 1. Comparison of Gait Speed between the Group A and B. 


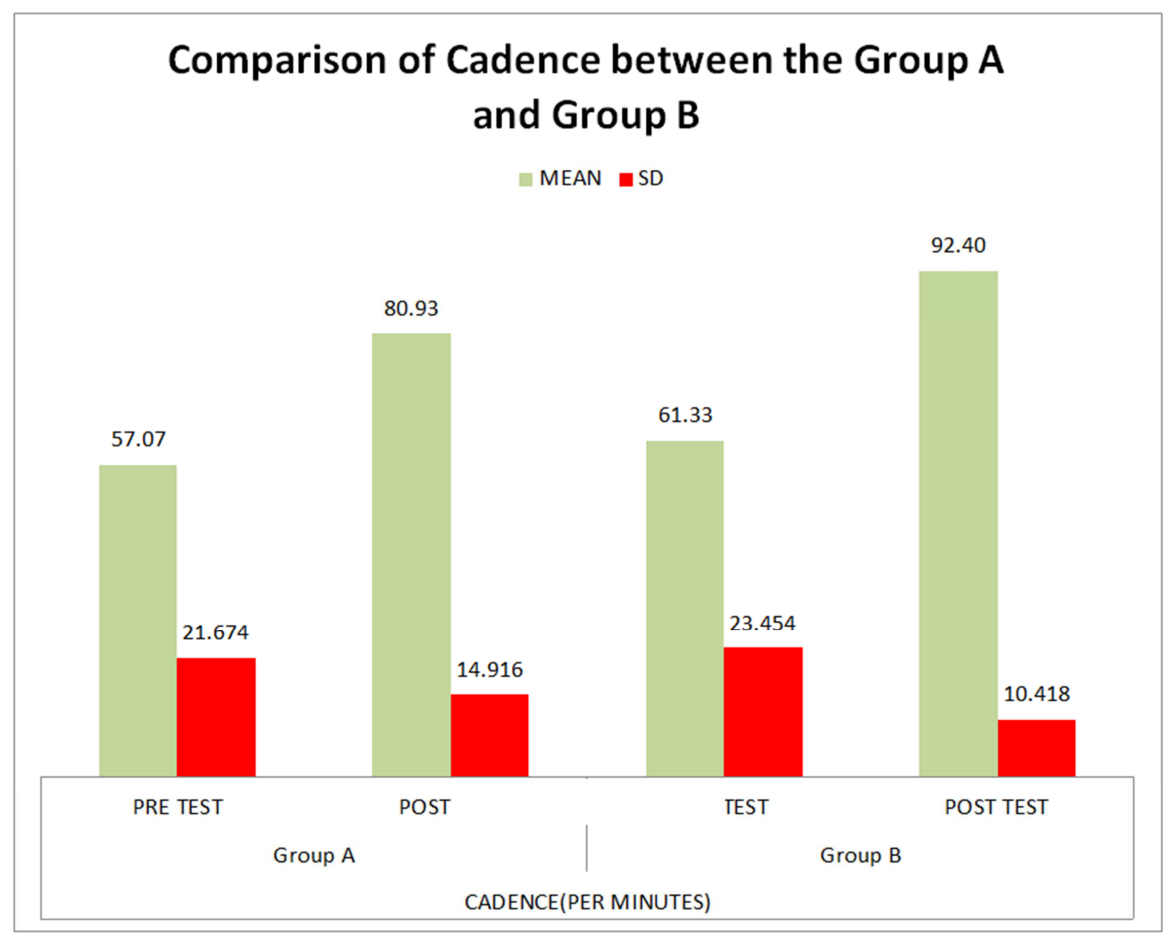

Figure 2. Comparison of Cadence between the Group A and Group B.

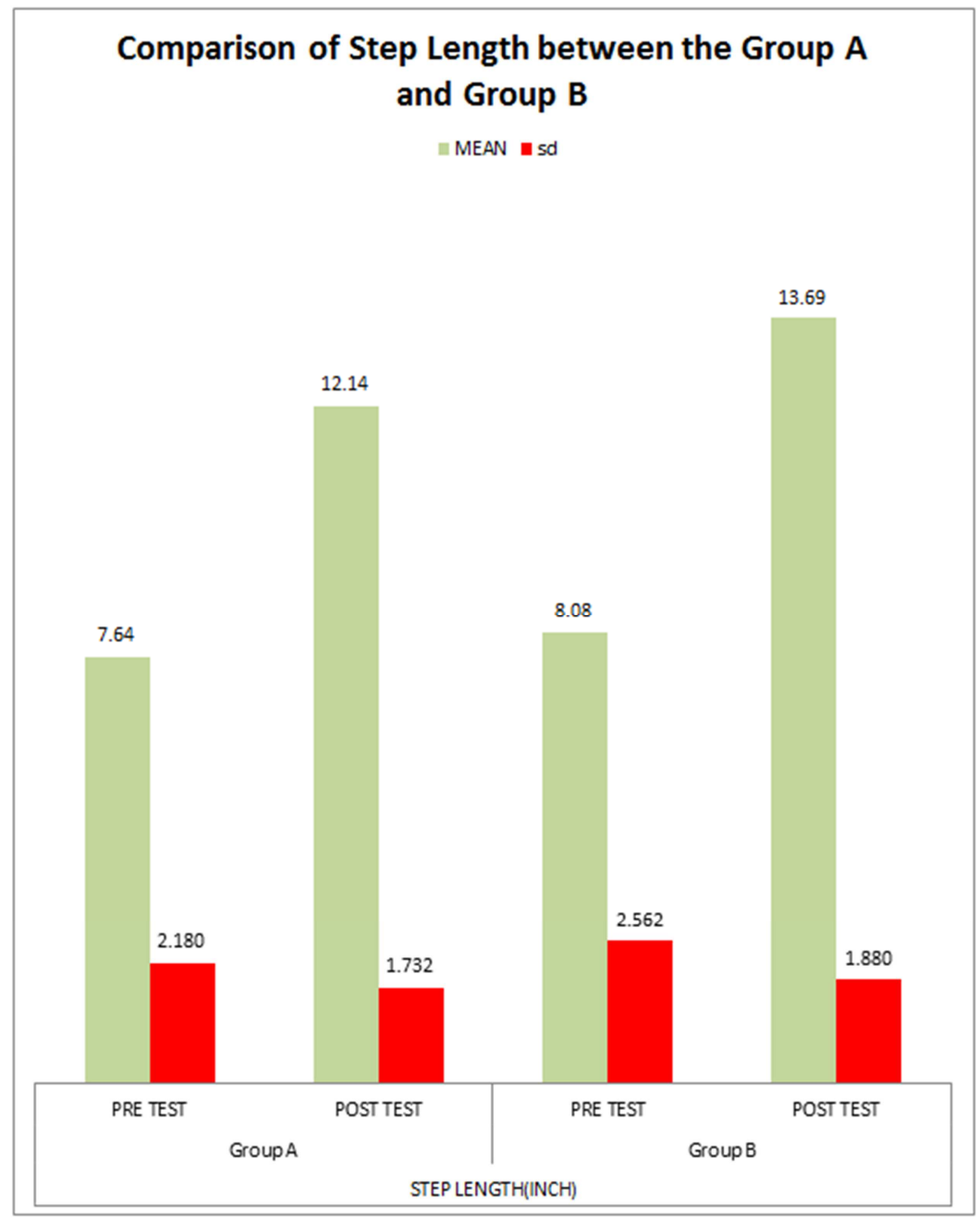

Figure 3. Comparison of Step Length between the Group A and Group B. 


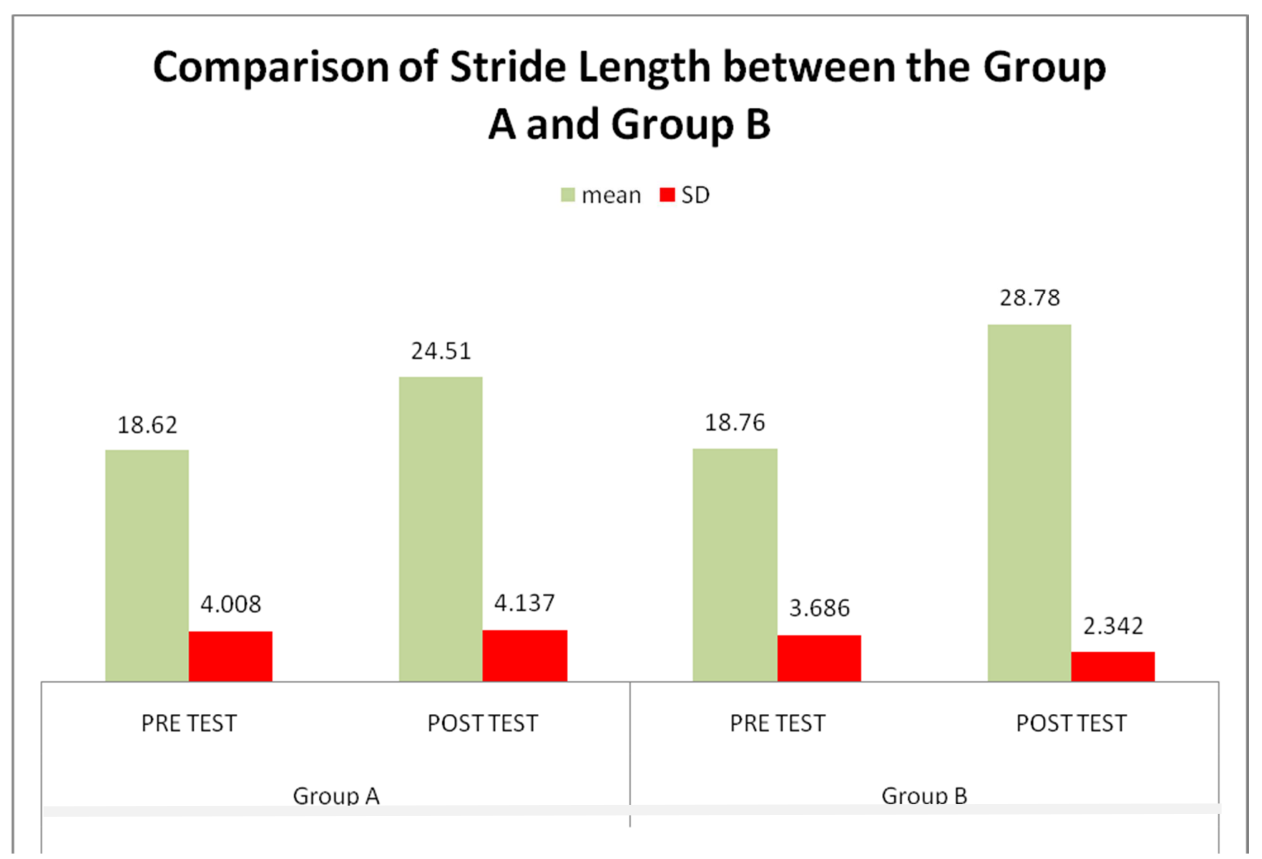

Figure 4. Comparison of Stride Length between the Group A and Group B.

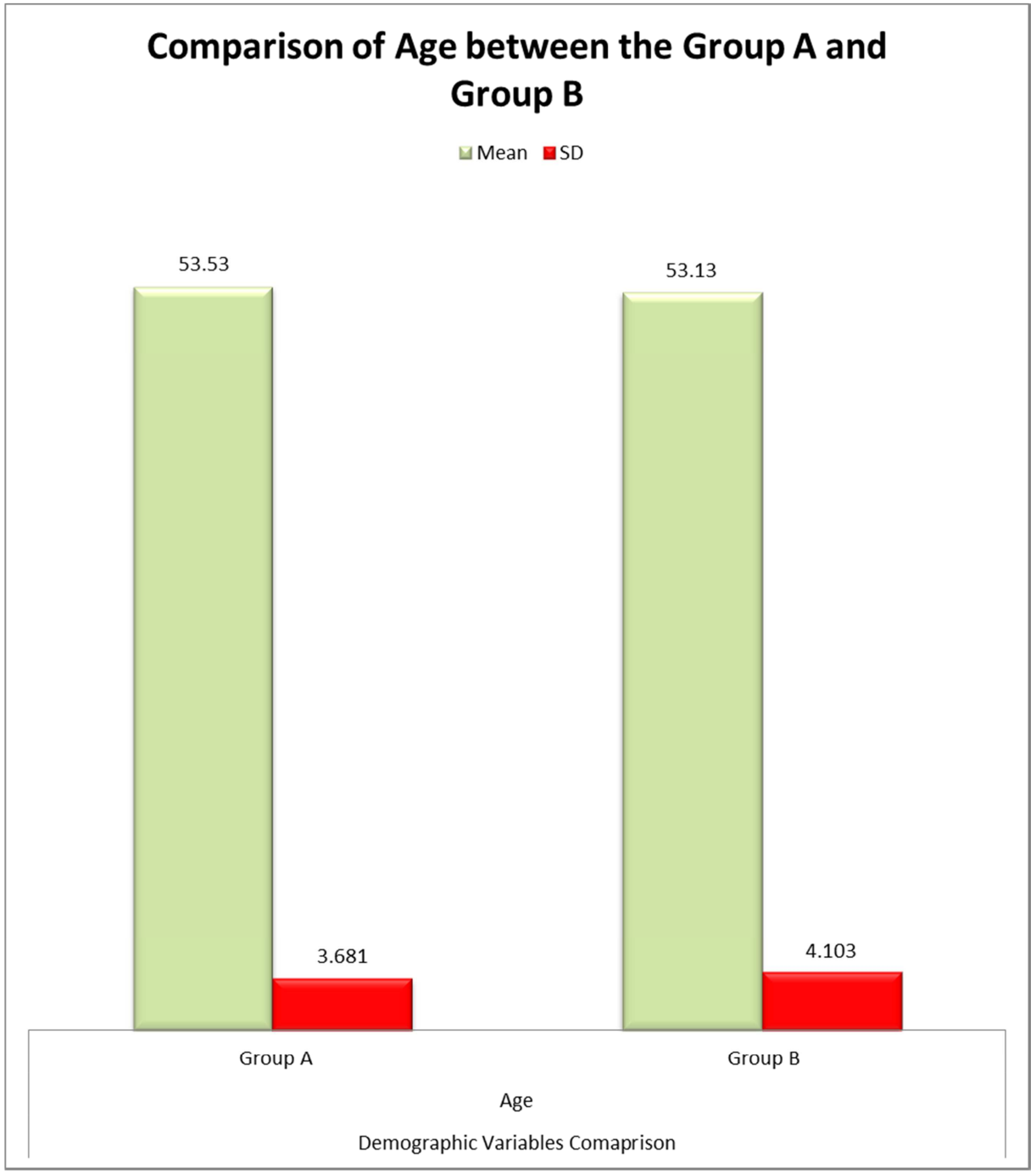

Figure 5. Comparison of Age between the Group A and Group B. 
Table 1. Comparison of Gait Speed between the Group A and Group B.

\begin{tabular}{llll}
\hline \multirow{2}{*}{ UnPaired 't' test } & \multicolumn{2}{l}{ Baseline (Pre) Readings Comparison } & Post Readings Comparison \\
\cline { 2 - 4 } & Gait Speed & Group B & Gait Speed \\
\cline { 2 - 3 } & Group A & 0.37 & 0.52 \\
\hline Mean & 0.37 & 0.119 & 0.140 \\
Standard Deviation & 0.104 & & 2.276 \\
't' value & 0.114 & 2.05 & 0.63 \\
$t_{0.05}$ & & & Significant \\
Results & Non-Significant & & 0.126 \\
\hline
\end{tabular}

$\mathrm{p}>0.05$ Non Significant.

$\mathrm{p}<0.05$ Significant.

Table 2. Comparison of Cadence between the Group A and Group B.

\begin{tabular}{llll}
\hline \multirow{2}{*}{ UnPaired 't' test } & \multicolumn{2}{l}{ Baseline (Pre) Readings Comparison } & Post Readings Comparison \\
\cline { 2 - 3 } & Cadence & Cadence \\
\cline { 2 - 3 } & Group A & Group B & Group A \\
\hline Mean & 57.07 & 61.33 & 80.93 \\
Standard Deviation & 21.674 & 23.454 & 14.916 \\
't' value & 0.517 & & 2.441 \\
$t_{0.05}$ & & 2.05 & Group B \\
Results & Non-Significant & & Significant \\
\hline
\end{tabular}

$\mathrm{p}>0.05$ Non Significant.

$\mathrm{p}<0.05$ Significant.

Table 3. Comparison of Step Length between the Group A and Group B.

\begin{tabular}{llll}
\hline \multirow{2}{*}{ UnPaired ' $t$ ' test } & \multicolumn{2}{l}{ Baseline (Pre) Readings Comparison } & Post Readings Comparison \\
\cline { 2 - 3 } & Step Length & Step Length \\
\cline { 2 - 3 } & Group A & Group B & Group A \\
\hline Mean & 7.64 & 8.08 & 12.14 \\
Standard Deviation & 2.180 & 2.562 & 1.732 \\
' $\mathrm{t}$ ' value & 0.507 & & 2.353 \\
$\mathrm{t}_{0.05}$ & & 2.05 & 13.69 \\
Results & Not-Significant & & Significant \\
\hline
\end{tabular}

$\mathrm{p}>0.05$ Non Significant.

$\mathrm{p}<0.05$ Significant.

Table 4. Comparison of Stride Length between the Group A and Group B.

\begin{tabular}{|c|c|c|c|c|}
\hline \multirow{3}{*}{ UnPaired ' $t$ ' test } & \multicolumn{2}{|c|}{ Baseline (Pre) Readings Comparison } & \multicolumn{2}{|c|}{ Post Readings Comparison } \\
\hline & \multicolumn{2}{|c|}{ Stride Length } & \multicolumn{2}{|c|}{ Stride Length } \\
\hline & Group A & Group B & Group A & Group B \\
\hline Mean & 18.62 & 18.76 & 24.51 & 28.78 \\
\hline Standard Deviation & 4.008 & 3.686 & 4.137 & 2.342 \\
\hline 't' value & 0.100 & & 3.476 & \\
\hline & & 2.05 & & \\
\hline Results & Not-Significant & & Significant & \\
\hline
\end{tabular}

$\mathrm{p}>0.05$ Non Significant.

$\mathrm{p}<0.05$ Significant.

Table 5. Comparison of Age between the Group A and Group B.

\begin{tabular}{lll}
\hline \multirow{2}{*}{ UnPaired ' $t$ ' test } & Age & Group B \\
\cline { 2 - 3 } & Group A & 53.13 \\
\hline Mean & 53.53 & 4.103 \\
Standard Deviation & 3.681 & \\
' $\mathrm{t}$ ' value & 0.281 & \\
$\mathrm{t}_{0.05}$ & 2.05 & \\
Results & Not Significant & \\
\hline
\end{tabular}

$\mathrm{p}>0.05$ Non Significant.

$\mathrm{p}<0.05$ Significant. 


\section{Discussion}

Gait is a complex process and stroke patients have a characteristic gait pattern. Thus, gait speed assessment can be used as a criterion of functional recovery for stroke patient. The standard of independent gait ability and increase in gait speed are the final steps of stroke rehabilitation, providing the chance for stroke patients to return to their community and actively participate in social activities. [19] In this study, The data was analyzed through Paired ' $t$ ' test for comparison within the dual task training (Group A), which gave ' $\mathrm{t}$ ' value for gait speed8.095, cadence 9.994, step length 8.768, stride length 6.285 , which was statistically significant andtask oriented training (Group B), which gave ' $t$ ' value for gait speed 10.724, cadence 6.094, step length 6.618, stride length 7.988 which was statistically significant. There after unpaired ' $t$ ' test was applied for comparison between Group A and Group B which gave ' $t$ ' value for gait speed is 2.276, cadence 2.441 , step length 2.353 , stride length 3.476 which were statistically significant. This above values showed that after intervention there was significant improvement within both the Groups i.e dual task training and task oriented training but when we compare both groups task oriented training has shown more improvement in gait of chronic stroke patients than the dual task training. Irrespective to the technique both groups showed significant improvement in gait which can be explained on the basis of phenomena of neural plasticity following brain lesion, supported by various studies on brain plasticity. [18] Repetitive exercises and training in real life task following stroke may be a critical stimulus for the making of new more effective functional connections within remaining brain tissue. Training and practice using methods that facilitate motor relearning would be essential to the formation of new functional connections. Neural system is inherently flexible adaptive and responding according to many factors like patterns of use. The complex organization provides the foundation for functional plasticity in motor cortex. Cortical representation reflects changes associated with skill development and provoked by active, repetitive, training and practice. So it can be because of specificity of training with respect to different environmental conditions as practiced in both the techniques showed improvement in gait. [19]

Task oriented training is based on the motor learning theory. [20] According to this theory, for doing any task attention is directed to the desired outcome and critical task elements. This helps the learner to develop an internal cognitive map or reference of correctness. Task oriented training prevents learned nonuse of the involved segments while stimulating central nervous system recovery. [21] Learning comes from an interaction and strengthening among multiple systems and there may be strong neural connections between related systems that can be crudely viewed as representations. This internal representation needs to be matched to the external environment and functional movement likely emerges as a result of this interaction. [22] Motor learning involves a very important component of analysis of task before actually performing it. Tasks are broken down in to discrete parts during analysis. [21] This analysis leads to in depth cognitive processing of the task leading to enhanced functional connections in the motor cortex. [19] These functional connections in motor cortex helps in learning process that depends on length of time given to the task during practice, the nature of the task, prior experience, motivation of the learner \& feedback. [21] Stronger perceptual trace developed in motor cortex through practice improves capability of learner to use close loop process for learning movements required during gait cycle, in turn improve gait parameters.

Task oriented training involves various tasks which are representation of real life activitities which are practiced on day to day basis, this practice leads to more enhanced cognitive map in the motor cortex. [19] In the study of task oriented purposed that outcomes of a task are dependent on principles of specificity, variability, repetition and intensity. The principle of variability and specificity leads to overall improvement in performance of task, as it gives an opportunity to the person to practice a task in the variety of contexts. Hence improvement in retention of skills required for a specific activity, which is gait in this study. [23] The reason which can be attributed for significant improvement in dual task group might be that, dual task training, provides information regarding restoration of automatism of balance control by influencing the reorganization process of central nervous system with respect to postural stability. [24] Dual task training is based on the dual task interference theory. Lateral prefrontal cortical structures are recruited when dual tasking involves more serial response selection, whereas striatal structures of the basal ganglia are recruited when there is a more parallel response selection process. The role of basal ganglia is recognized in the model of prioritization for dual task interference which plays important role in habitual responses (i.e well practiced and more automated tasks that require relatively little attention resources) which dominate in situation where one must act quickly and that habitual responses are characterized by parallel processing. [10] Dual task the internal focus of attention, concentrating on the movement itself, during the performance of a motor task inhibits self-operating postural control due to conscious control of the posture, while the external focus of attention, concentrating on the result of the movement during the motor task, promotes self operating postural control. Hence while perfoming a dual task both of internel and external focus of attention determines the final outcome of the task. [9] Dual task performance requires a high level of concentration, which results in decreased gait ability in stroke patients. Structural problems within the frontal lobe and motor areas resulting from a stroke result in reduced concentration and increased cognitive motor interference. Consequently, concentration during performance of dual tasks is directed towards the cognitive component of the task while concentration on other task components decreases. [8] Comparison when done between both the groups task oriented training comes out to be better in improving various 
outcomes of the gait. This result could be explained on the basis of Task Automatization Hypothesis, which states that practicing only one task at a time allows participants to automatize the performance of individual tasks. As a result, the processing demand required to perform the tasks is decreased, leading to a more rapid development of skills. [25] Reason which may be attributed for task oriented training group to show significant improvement is that subjects might have concentrated only on the task that were performed by them. Full attention was given to the task only.

To minimize the changes and to promote recovery, extended practice requiring the patient's active participation is necessary. Task oriented training incorporates practice that is supervised and structured to involve functional goals and realistic tasks which could be the link factor for better outcomes of task oriented training. [11] This attempt of comparison among the two techniques suggest task oriented training programme to be a better Technique for improving gait parameters in chronic stroke. This current study would provide a justification that task oriented training programme could be one of the efficient treatment technique for improving the gait in chronic stroke.

\section{Conclusion}

This study concluded that there was significant difference between the effect of dual task and task oriented training programme on gait in chronic stroke. Pre treatment data was taken using gait parameters i.e gait speed, cadence, step length, stride length as outcome measures. Group A performed dual task training and group B performed task oriented training. Post treatment data was collected and analysed. But there were some drawbacks likesmall sample size, all gait parameters were not assessed and only middle age group were considered. Stroke is one of the main neurological conditions which affect the gait pattern but there are many other neurological conditions with Gait pathology other than stroke which can be considered for seeing the effect of dual task and task oriented technique.

\section{Abbreviation}

$\begin{array}{ll}\text { WHO } & \text { World Health Organization } \\ \text { DT } & \text { Dual Task } \\ \text { MMSE } & \text { Mini -Mental State Examination } \\ \text { MAS } & \text { Modified Ashworth Scale } \\ \text { MCDGT } & \text { Motor and Cognitive Dual Task Gait Training } \\ \text { MDGT } & \text { Motor Dual Task Gait Training } \\ \text { CDGT } & \text { Cognitive Dual Task Gait Training }\end{array}$

\section{References}

[1] Minino, A. M., Xu, J., Kochanek, K. D. (2011). "Deaths: final data for 2008. National Vital Statistics Reports. Atlanta: National Center for Health Statistics, Center for Disease Control and Prevention" 59 (10), pp. 1-126.
[2] Obembe, A. O., Olaogun, M. O. B., \&Adedoyin, R. A. (2012). "Differences in Gait between hemorrhagic and ischemic Stroke survivors", Journal of Medicine and Medical Sciences. 3 (9) pp. 556-561.

[3] Strandgaard, S., \& Paulson, O. B. (1990). "Pathophysiology of Stroke", Journal of Cardiovascular Pharmacology. 15 (1) pp. S38-S42.

[4] Belda-Lois, J. M., Del-Horno, S. M., Bosch, I. B., Moreno, J. C., Pons, J. L., et al. (2011). "Rehabilitation of Gait after Stroke: A review towards a top down approach", Journal of Neuroengineering and Rehabilitation. 8 (66) pp. 1-19.

[5] Bowen, A., Wenman, R., Mickelborough, J., Foster, J et al. (2001). "Dual Task effects of walking on velocity and balance following a Stroke", Age and Aging. 30 pp. 319-323.

[6] Ferrarello, F., Baccini, M., Rinaldi, L. A., et al. (2011). "Efficacy of physiotherapy interventions late after stroke: a meta-analysis", Journal of neurology, neurosurgery and psychiatry. 82 pp. 136-143.

[7] Rai, R. K., Arora, L., Sambyal, S., \&Arora, R. (2014). "Efficacy of Trunk Rehabilitation and balance training on trunk control, Balance and Gait in Post Stroke hemiplegic patients: A Randomized Controlled Trial”, Journal of Nursing and Health Science. 3 (3) pp. 27-31.

[8] Lee, G. C.,\& Choi, W. J. (2012). "The effects of dual task training on ambulatory abilities of stroke patients: Review of the latest trend", Physical Therapy Rehabilitation Science. 1 (1) pp. 1-5.

[9] Liu, Y. C., Yang, Y. R., Tsai, Y. A., Wang, R. Y. (2017). "Cognitive and motor dual task gait training improve dual task gait performance after stroke - A randomized controlled pilot trial". 7 (1). doi: 10.1038/s41598-017-04165-y

[10] Plummer, P., Villalobos, R. M., Vayda, M. S., Moser, M.,\& Johnson, E. (2014). "Feasibility of Dual Task Gait Training for Community Dwelling Adults after Stroke: A Case Series', Stroke Research and Treatment" pp. 1-12.

[11] Outermans, J. C., van Peppen, R. P., Wittink, H., Takken, T., Kwakkel, G. (2010). "Effects of a high-intensity task-oriented training on gait performance early after stroke: a pilot study. Clinical rehabilitation". 24 (11) pp. 979-87.

[12] Liu, T. W., Ng, G. Y., Chung, R. C., Ng, S. S. (2019). "Decreasing fear of falling in chronic stroke survivors through cognitive behavior therapy and task-oriented training. Stroke". 50 (1) pp. 148-54.

[13] Wevers, L., Port, I. V., Vermue, M., Mead, G., \&Kwakkel, G. (2009). "Effects of Task Oriented Circuit class Training on walking competency after Stroke", stroke. 40 pp. 2450-2459.

[14] Vats, M. (2013), "Efficacy of Task specific Step Up Exercises on the Gait parameters of Chronic Hemiparetic Stroke Individuals", International Journal of Physiotherapy and Research, 1 (4) pp. 130-37.

[15] Kim, B. H., Lee, S. M., Bae, Y. H., Yu, J. H., \& Kim, T. H. (2012) "The Effect of Task Oriented Training on Trunk control ability, Balance and Gait of Stroke patients", Journal of Physical Therapy Science, 24 (6) pp. 519-522.

[16] An, H. J., Kim, J. I., Kim, Y. R., et al. (2014). "The effect of Various dual task training Methods with Gait on the Balance and Gait of Patients with Chronic Stroke", Journal ofPhysical Therapy Science. 26 (8) pp. 1287-1291. 
[17] Shim, S., Yu, J., Jung, J., Kang, H., \& Cho, K. (2012), “Effects of Motor Dual Task Training on Spatiotemporal Gait parameters of Post Stroke patients", Journal of Physical Therapy Science, 24 (9) pp. 845-848.

[18] Thielman, G. T., Dean, C. M., \& Gentile, A. M. (2004). "Rehabilitation of reaching after Stroke: Task related training versus Progressive resisted exercises", Archives of Physical Medicine and Rehabilitation. 85 pp. 1613-1618.

[19] Kanase, S. B., \&Varadharajulu, G. (2012), "Effect of Task Related Training versus Conventional Training on Walking Performances in Post Stroke Patients", International Journal of Science and Research. 3 (11) pp. 2786-2790.

[20] Song, H. S., Kim, J. Y., \& Park, S. D. (2015) "Effect of the Class and individual applications of Task Oriented circuit Training on gait ability in patients with Chronic Stroke", Journal of Physical Therapy Science. 27 (1) pp. 187-189.

[21] O'Sullivan, S. B., \& Schmitz, J. T. (2007), "Physical Rehabilitation", edt. $5^{\text {th }}$, Philadelphia.
[22] Muratori, K. M., Lamberg, E. M., Quinn, L., \& Duff, S. V. (2013) "Applying principles of motor learning and control to upper extremity rehabilitation", Journal ofHand Therapy. 26 (2) pp. 94-103.

[23] Straudi, S., Martinuzzi, C., Pavarelli, C et al. (2014) "A task oriented circuit training in multiple sclerosis: a feasible study", Journal of Biomedicine Central. 14 (124) pp. 1-9.

[24] Khan, M., Prathap, S., \&Kumerasan. (20130, "A study to compare the effect of cognitive dual task versus motor dual task on balance training in stroke patients", International Journal of Biology, Pharmacy and Allied Sciences. 2 (9) pp. 1769-1776.

[25] Silsupadol, P., Shumway-Cook, A., Lugade, V et al. (2009), "Effects of Single Task Versus Dual Task Training on Balance Performance in Older Adults: A Double-Blind, Randomized Controlled Trial", Archives of Physical Medicine and Rehabilitation, 90 (3) pp. 381-387. 